\title{
Voice Outcome in Patients Treated With Endoscopic Laryngopharyngeal Surgery for Superficial Hypopharyngeal Cancer
}

\author{
Ichiro Tateya $^{1} \cdot$ Shuko Morita $^{2} \cdot$ Seiji Ishikawa $^{1} \cdot$ Manabu Muto $^{3} \cdot$ Shigeru Hirano $^{1} \cdot$ Yo Kishimoto $^{1}$ \\ Nao Hiwatashi ${ }^{1} \cdot$ Juichi Ito ${ }^{1}$ \\ Departments of ${ }^{1}$ Otolaryngology-Head \& Neck Surgery, ${ }^{2}$ Gastroenterology \& Hepatology, and ${ }^{3}$ Clinical Oncology, \\ Graduate School of Medicine, Kyoto University, Kyoto, Japan
}

Objectives. Endoscopic laryngopharyngeal surgery (ELPS) is a minimally invasive transoral surgery that was developed to treat superficial larygo-pharyngeal cancer, in which a mucosal lesion is resected transorally while preserving deeper structures by subepithelial injection. The purpose of this retrospective study is to evaluate voice outcome in patients who underwent ELPS for superficial hypopharyngeal cancer. As important structures in producing voice, such as intrinsic laryngeal muscles, their fascia, and recurrent laryngeal nerve, are located in the medial side of the piriform sinus and the postcricoid region of the hypopharynx, we focused on patients with cancer lesions involving these regions.

Methods. From April 2010 to March 2011, 25 consecutive patients with superficial laryngopharyngeal cancer were treated with ELPS at Kyoto University Hospital. Among the 25 patients, 11 patients with cancer lesions on the medial side of the piriform sinus or the postcricoid area were studied. Preoperative and postoperative voice functions including maximum phonation time (MPT), mean flow rate (MFR), jitter, shimmer, soft phonation index (SPI), and noise-toharmonic ratio (NHR), were compared retrospectively.

Results. Five of 11 cancer lesions had submucosal invasion and no lesion had invaded the muscular layer pathologically. T stage was classified as Tis in 5 cases, T1 in 4 cases, and T2 in 2 cases. All lesions involved the medial side of the piriform sinus and 2 also involved the postcricoid area. Vocal fold movement was normal in all cases after the surgery. Average preoperative and postoperative values for MPT, MFR, jitter, shimmer, SPI, and NHR, were 22.7 seconds and 23.4 seconds, $165 \mathrm{~mL} / \mathrm{sec}$ and $150 \mathrm{~mL} / \mathrm{sec}, 1.53 \%$ and $1.77 \%, 3.82 \%$ and $5.17 \%, 35.5$ and 36.6 , and $0.13 \%$ and $0.14 \%$, respectively. There was no statistical difference between preoperative and postoperative data for all values examined.

Conclusion. ELPS is useful in preserving voice function in the treatment of superficial hypopharyngeal cancer. Preserving the deeper structures including intrinsic muscles and their fascia may be important for preserving voice function as long as the lesions are superficial.

Keywords. Phonation; Laryngeal Muscles; Narrow Band Imaging

- Received November 29, 2014

Revised January 15, 2015

Accepted March 11, 2015

- Corresponding author: Ichiro Tateya

Department of Otolaryngology-Head \& Neck Surgery, Graduate School of

Medicine, Kyoto University, Sakyo-ku, Kyoto 606-8507, Japan

Tel: +81-75-751-3346, Fax: +81-75-751-7225

E-mail: tateya@ent.kuhp.kyoto-u.ac.jp

\section{INTRODUCTION}

Narrow band imaging (NBI) is an innovative optical technology that can increase contrast showing precise morphological changes in the mucosal surface. When combined with magnifying endoscopy, NBI allows clear visualization of microvascular structures [1]. In the head \& neck region, we have previously reported

Copyright (C) 2016 by Korean Society of Otorhinolaryngology-Head and Neck Surgery.

This is an open-access article distributed under the terms of the Creative Commons Attribution Non-Commercial License (http://creativecommons.org/licenses/by-nc/4.0)

which permits unrestricted non-commercial use, distribution, and reproduction in any medium, provided the original work is properly cited. 
that magnifying gastrointestinal endoscopy with the NBI function (ME-NBI) provides high-resolution images and is useful in detecting early superficial laryngopharyngeal cancers, which are difficult to detect by standard endoscopy [2-4]. We have been treating such lesions with endoscopic laryngopharyngeal surgery (ELPS). ELPS is a minimally invasive transoral surgery that was developed to treat superficial laryngopharyngeal cancer [5,6], in which a mucosal lesion is resected transorally while preserving deeper structures by subepithelial injection.

The purpose of this study is to evaluate voice outcome in patients who underwent ELPS for superficial hypopharyngeal cancer. Structures important in producing voice, such as intrinsic laryngeal muscles, their fascia, and the recurrent laryngeal nerve, are located in the medial side of the piriform sinus and the post-

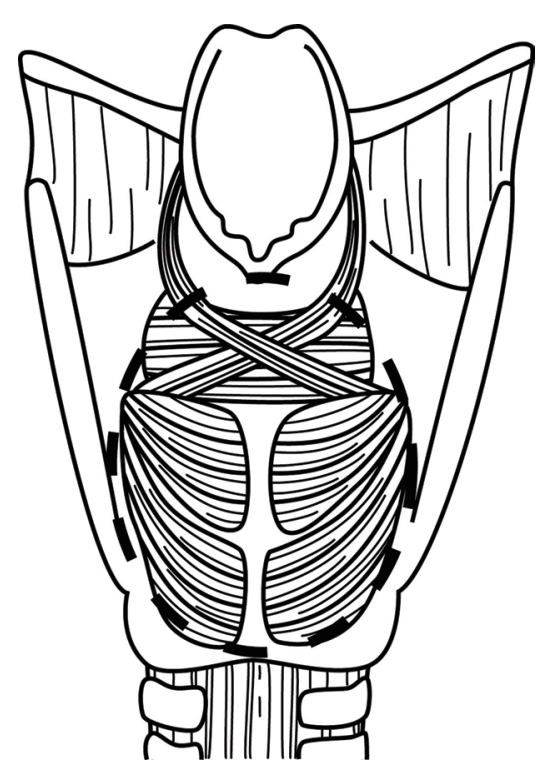

Fig. 1. Schematic views of the medial side of the piriform sinus and the postcricoid area. Cancer lesions involving the area (broken line) were involved in this study.
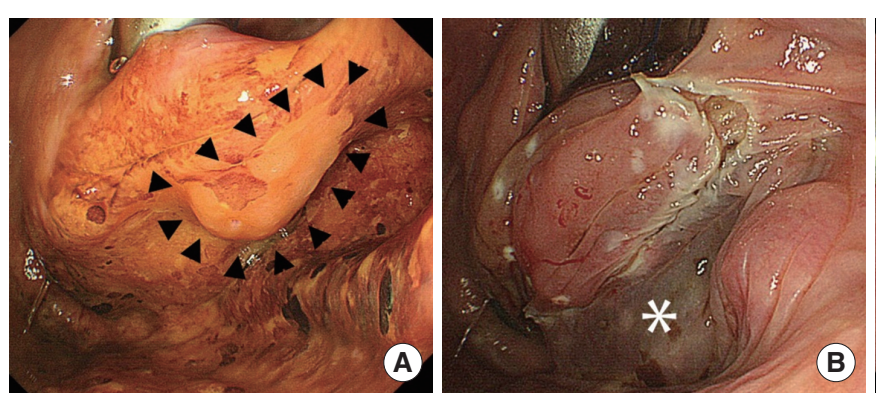

cricoid area of the hypopharynx. The risk of affecting voice function should be considered, especially when the lesion involves these "vital" regions for producing voice. Thus, we focused on patients with a cancer lesion involving the medial side of the piriform sinus or postcricoid region, and retrospectively examined their preoperative and postoperative voice function.

\section{MATERIALS AND METHODS}

\section{Patients}

During the period from April 2010 to March 2011, 25 consecutive patients with superficial laryngopharyngeal cancer were treated with ELPS under general anesthesia at Kyoto University Hospital. Among the 25 patients, 11 patients who had cancer lesions involving the medial side of the piriform sinus or the postcricoid area (Fig. 1) were studied. Written informed consent for the treatment was obtained from all patients, and this study was approved by the institutional review board of the Graduate School of Medicine, Kyoto University.

Histological diagnosis of the lesions was made in accordance with the World Health Organization classification of head and neck tumors. Evaluation of tumor invasion was made according to the general rules for clinical studies of head and neck cancer by the Japanese Society for Head and Neck Cancer [7] and the Japanese classification of esophageal cancer by the Japan Esophageal Society [8]. If the lesion was diagnosed as carcinoma in situ (CIS) or carcinoma with invasion to the subepithelial layer (not to the muscular layer), ELPS was indicated as a minimallyinvasive treatment. All lesions were detected by NBI with a magnifying endoscope and histologically confirmed by examination biopsy specimens as high grade dysplasia/CIS or squamous cell carcinoma.

\section{Surgical procedure (Fig. 2)}

The concept of ELPS is the same as that of endoscopic submu-
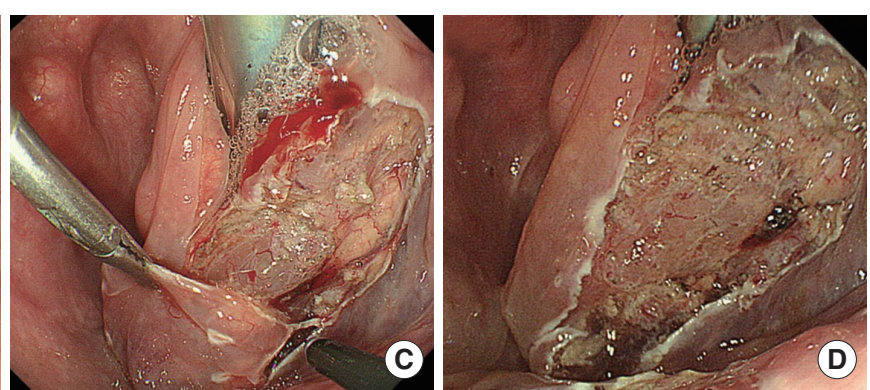

Fig. 2. Endoscopic laryngopharyngeal surgical procedure. (A) The cancer lesion identified as an unstained area by iodine staining (arrow heads) is located in the medial side of the right piriform sinus. The lesion is identified as an unstained area by iodine staining. (B) Subepithelial injection $\left(^{*}\right)$ is performed to lift the lesion above the surrounding mucosa and to create a safety space. Creating the safety space facilitates removal of the lesion and minimizes damage to the deep layers of the laryngopharyngeal wall, such as intrinsic laryngeal muscle, recurrent laryngeal nerve and its branch. (C) The lesion is dissected with a curved laryngeal forceps and a curved electrosurgical needle knife (Olympus Medical Systems, Tokyo, Japan). (D) The lesion was dissected and the fascia of the intrinsic laryngeal muscle and the recurrent laryngeal nerve were preserved. 
cosal dissection (ESD) in that both can perform en bloc resection of a cancer lesion following submucosal injection [6]. However, it differs from ESD in that the resection procedure is performed by a head and neck surgeon with both hands. In short, under general anesthesia, a curved rigid laryngoscope (Nagashima Medical Instruments Co., Tokyo, Japan) is inserted to provide a working space in the pharyngeal lumen and a ME-NBI system (GIF TYPE H260Z; Olympus Medical Systems, Tokyo, Japan) is inserted transorally by a gastroenterologist to visualize the surgical field. Tumor resection was performed by a head and neck surgeon with the assistance of a gastroenterologist. A solution comprised of a mixture of epinephrine $(0.02 \mathrm{mg} / \mathrm{mL})$ and saline is injected into the subepithelial layer beneath the lesion, so that the lesion can be lifted above the surrounding mucosa and a safety space created. Creating the safety space facilitates tumor removal and minimizes damage to the deep layers of the laryngopharyngeal wall, such as intrinsic laryngeal muscles, their fascia, the recurrent laryngeal nerve and its branches, and the internal branch of the superior laryngeal nerve. A circumferential incision into the submucosa was then created around the lesion with a curved electrosurgical needle knife (Olympus Medical Systems) and the lesion is dissected from the pharyngeal wall.

\section{Voice function and statistical analysis}

Voice function was evaluated by analyzing the maximum phonation time (MPT), mean flow rate (MFR), jitter (\%), shimmer (\%), soft phonation index (SPI), and noise-to-harmonic ratio (NHR), preoperatively and postoperatively. Postoperative analysis was done a median of 87 days after surgery. The MFR was evaluated with PA-1000 (Nagashima Co., Tokyo, Japan). The vocal signal was recorded with a Kay Computer Speech Lab 4300B supported by a personal computer including a Shure-Prolog SM48 microphone. Analysis of a voice sample, directly recorded using digital technology and with a sample frequency of $50 \mathrm{kHz}$, was performed using the multi-dimensional voice program (MDVP ver. 2.3, Kay Elemetrics Corp., Lincoln Park, NJ, USA), and jitter, shimmer, SPI, and NHR were evaluated.

Wilcoxon signed rank test was used for statistical analysis to compare preoperative and postoperative data in each patient.
Differences at $P<0.05$ were regarded as statistically significant.

\section{RESULTS}

\section{A representative case}

A 69-year-old male underwent ELPS for superficial hypopharyngeal cancer spreading over one third of the postcricoid area, the entire right preform sinus, the entire posterior wall, and one third of the entrance of the esophagus. About two thirds of the hypopharyngeal mucosa was resected (Fig. 3). Eleven months after surgery, the vocal fold movement was normal on the bilateral side and there was no glottal gap during phonation even though the right piriform sinus was totally obstructed (Fig. 3). MPT and MFR were 22 seconds (normal, >12 seconds) and 107 $\mathrm{mL} / \mathrm{seconds}$ (normal range, 80 to $190 \mathrm{~mL} / \mathrm{sec}$ ), respectively.

\section{Lesion characteristics and voice function}

Regarding the pathology, 5 of the 11 specimens were CIS. The rest 6 specimens included four $\mathrm{T} 1$ lesions and two T2 lesions. These 6 lesions showed subepithelial invasion, but did not invade to the muscular layer. Surgical margin was negative in all cases.

In all cases, the medial side of the piriform sinus was involved and in 2 cases the postcricoid area was involved. Vocal fold movement was normal in all cases after the surgery.

Summary of the preoperative and postoperative data on MPT, MFR, jitter, shimmer, SPI, and NHR (normal range, <0.190) are shown in Fig. 4. One case with Tis lesion in the piriform sinus showed increased jitter and shimmer values postoperatively, from $2.454 \%$ to $4.814 \%$, and from $2.213 \%$ to $11.317 \%$, respectively. However, MPT and MFR of the case showed no change postoperatively, from 12 seconds to 12 seconds, and from $220 \mathrm{~mL} / \mathrm{sec}$ to $206 \mathrm{~mL} / \mathrm{sec}$, respectively. Average preoperative and postoperative values for MPT, MFR, jitter (normal range, $<1.04 \%$ ), shimmer (normal range, $<3.81 \%$ ), SPI (normal range, $<14.12$ ), and NHR (normal range, $<0.19$ ), were 22.7 seconds and 23.4 seconds, $165 \mathrm{~mL} / \mathrm{sec}$ and $150 \mathrm{~mL} / \mathrm{sec}, 1.53 \%$ and $1.77 \%, 3.82 \%$ and $5.17 \%, 35.5$ and 36.6 , and $0.13 \%$ and
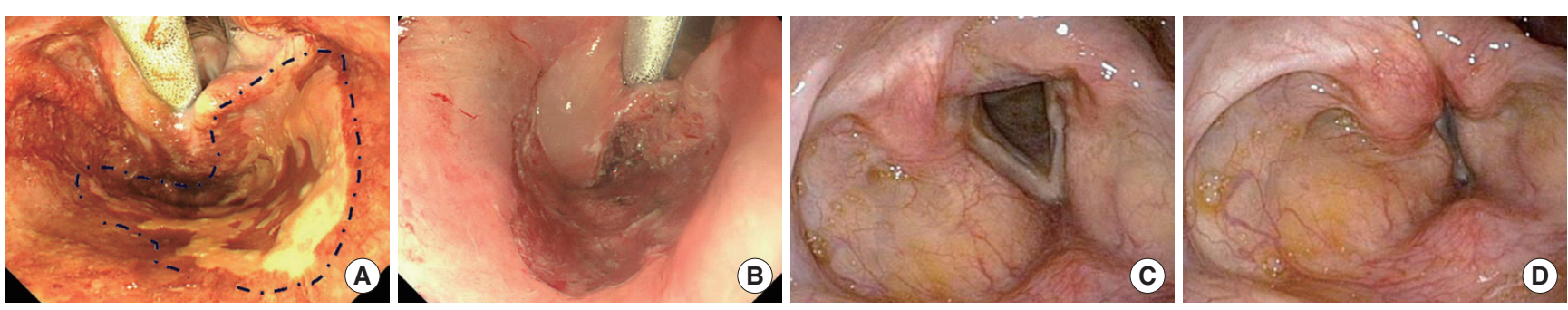

Fig. 3. Representative case. (A) Superficial hypopharyngeal cancer (broken lines) spreading over one third of the postcricoid area, the entire right preform sinus, the entire posterior wall, and one third of the entrance of the esophagus. (B) About two-thirds of the hypopharyngeal mucosa and one third of the entrance of the esophagus were resected. (C) Eleven months after surgery, the right piriform sinus was totally obstructed by scarring. (D) Vocal fold movement was normal in the bilateral side and there was no glottal gap during phonation despite scar formation in the right piriform sinus. 

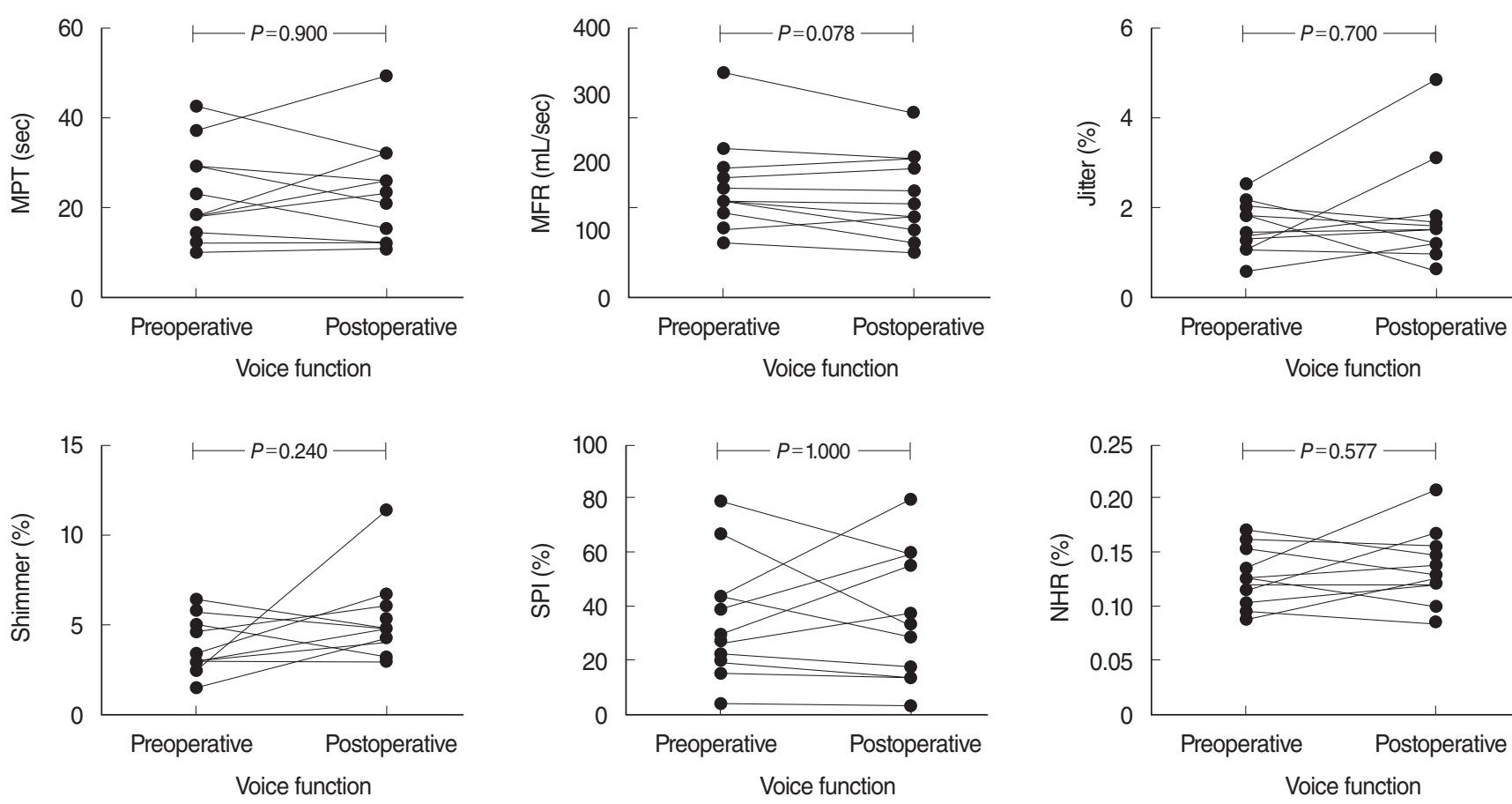

Fig. 4. Preoperative and postoperative voice function. Average preoperative and postoperative values for maximum phonation time (MPT), mean flow rate (MFR), jitter (normal range, <1.04\%), shimmer (normal range, <3.81\%), soft phonation index (SPI) (normal range, <14.12), and noise-to-harmonic ratio (NHR) (normal range, <0.190), were 22.7 seconds and 23.4 seconds, $165 \mathrm{~mL} / \mathrm{sec}$ and $150 \mathrm{~mL} / \mathrm{sec}, 1.53 \%$ and $1.77 \%, 3.82 \%$ and $5.17 \%, 35.5$ and 36.6 , and $0.13 \%$ and $0.14 \%$, respectively. P-values of statistical analysis (Wilcoxon signed rank test) in MPT, MFR, jitter, shimmer, SPI, and NHR were 0.900, 0.078, 0.700, 0.240, 1.000, and 0.577, respectively. There was no statistical difference between preoperative and postoperative data for all values examined.

$0.14 \%$, respectively. There was no statistical difference between preoperative and postoperative data in any of values examined.

\section{DISCUSSION}

Transoral surgery is a less invasive treatment that is becoming a major strategy for the treatment for laryngopharyngeal cancer. To date, several methods have been reported as less invasive transoral surgical approaches, such as transoral laser microsurgery [9], transoral robotic surgery (TORS) [10-12], and transoral videolaryngoscopic surgery [13]. These surgical methods have great advantages over conventional open surgery, especially in functional outcomes such as swallowing and use of voice, and are creating a paradigm shift in the treatment strategy for laryngopharyngeal cancer.

However, literature focused on details of voice outcomes following transoral surgery for hypopharyngeal cancer is very limited [14]. Park et al. [14] reported voice function in 23 patients who underwent TORS for hypopharyngeal cancer. Although NHR and shimmer had been maintained close to the normal range, fundamental frequency variation and jitter were increased due to decreased mobility of the ipsilateral vocal fold from scar formation in the piriform sinus.
The results of the present study suggest that ELPS for superficial hypopharyngeal cancer also maintains voice function even when the lesions involved "vital regions" for producing voice. Postoperative vocal fold movement is affected by several factors, which include not only damage to the recurrent laryngeal nerve, the arytenoid cartilage, and the intrinsic laryngeal muscles, such as lateral cricoarytenoid muscle, but also postoperative scar around the muscles and arytenoid cartilage. One of the main reasons why there was no significant impairment of voice function in this study is that all of the lesions resected were CIS or lesions which has no invasion to the musclular layer. In addition, from a technical point of view, we speculate that subepithelial injection during the procedure minimized excessive resection and contributed to reduce unnecessary damage to the nerves, muscles and especially their fascia, resulting in preserved movement of intrinsic laryngeal muscles despite scar formation in the epithelial layer. Keeping the deeper structures intact, especially the fascia of intrinsic laryngeal muscles, might contribute to preserve vocal fold movement in the piriform sinus. It must be mentioned that subepithelial injection may not be effective for lesions with muscular invasion because some of the intrinsic laryngeal muscles and their fascia must be resected with cancer when resecting invasive cancer.

That this is a retrospective study with a small sample size is a 
limitation. It is difficult to deny the possibility that no statistical difference in the analysis of this study may be due to the small sample size. Also, a comparative study between ELPS and other transoral approaches without subepithelial injection such as TORS has to be done in order to prove that vocal fold movement impairment due to scar contracture of muscles can be a mechanism of voice problem. A prospective comparative study with larger sample size will be necessary in the future to confirm these findings on voice outcome after ELPS for superficial hypopharyngeal cancer.

In conclusion, ELPS was useful in preserving voice function in the treatment of superficial hypopharyngeal cancer. Keeping the deeper structures intact, including the fascia of intrinsic muscles, may be important for preserving voice function when the lesions are superficial.

\section{CONFLICT OF INTEREST}

No potential conflict of interest relevant to this article was reported.

\section{ACKNOWLEDGMENTS}

This study was supported in part by grants-in-aid for research from the Ministry of Health, Labor, and Welfare of Japan. We would like to thank Ryuji Uozumi MS, Department of Biomedical Statistics and Bioinformatics, Kyoto University, for his help in the statistical analysis of this study.

\section{REFERENCES}

1. Muto M, Horimatsu T, Ezoe Y, Morita S, Miyamoto S. Improving vi- sualization techniques by narrow band imaging and magnification endoscopy. J Gastroenterol Hepatol. 2009 Aug;24(8):1333-46.

2. Muto M, Katada C, Sano Y, Yoshida S. Narrow band imaging: a new diagnostic approach to visualize angiogenesis in superficial neoplasia. Clin Gastroenterol Hepatol. 2005 Jul;3(7 Suppl 1):S16-20.

3. Muto M, Minashi K, YanoT, Saito Y, Oda I, Nonaka S, et al. Early detection of superficial squamous cell carcinoma in the head and neck region and esophagus by narrow band imaging: a multicenter randomized controlled trial. J Clin Oncol. 2010 Mar;28(9):1566-72.

4. Muto M, Nakane M, Katada C, Sano Y, Ohtsu A, Esumi H, et al. Squamous cell carcinoma in situ at oropharyngeal and hypopharyngeal mucosal sites. Cancer. 2004 Sep;101(6):1375-81.

5. Sato Y, OmoriY,Tagawa M.Treatment of superficial carcinoma in hypopharynx. Nihon Jibiinkoka Gakkai Kaiho. 2006 Dec;109(7):5816.

6. Tateya I, Morita S, Muto M, Miyamoto S, HayashiT, Funakoshi M, et al. Magnifying endoscope with NBI to predict the depth of invasion in laryngo-pharyngeal cancer. Laryngoscope. 2015 May;125(5): 1124-9.

7. Japan Society for Head and Neck Cancer. General rules for clinical studies on head and neck cancer. 5th ed. Tokyo: Kanehara Publishing Group; 2012.

8. Japan Esophageal Society. Japanese classification of esophageal cancer, tenth edition: part I. Esophagus. 2009 Mar;6(1):1-25.

9. Steiner W. Experience in endoscopic laser surgery of malignant tumours of the upper aero-digestive tract.Adv Otorhinolaryngol. 1988; 39:135-44.

10. O'Malley BW Jr, Weinstein GS, Snyder W, Hockstein NG. Transoral robotic surgery (TORS) for base of tongue neoplasms. Laryngoscope. 2006 Aug;116(8):1465-72.

11. Weinstein GS, O'Malley BW Jr, Snyder W, Hockstein NG. Transoral robotic surgery: supraglottic partial laryngectomy. Ann Otol Rhinol Laryngol. 2007 Jan;116(1):19-23.

12. Park YM, Byeon HK, Chung HP, Choi EC, Kim SH. Comparison study of transoral robotic surgery and radical open surgery for hypopharyngeal cancer. Acta Otolaryngol. 2013 Jun;133(6):641-8.

13. Tomifuji M, Araki K, Yamashita T, Shiotani A. Transoral videolaryngoscopic surgery for oropharyngeal, hypopharyngeal, and supraglottic cancer. Eur Arch Otorhinolaryngol. 2014 Mar;271(3):589-97.

14. Park YM, Kim WS, De Virgilio A, Lee SY, Seol JH, Kim SH.Transoral robotic surgery for hypopharyngeal squamous cell carcinoma: 3-year oncologic and functional analysis. Oral Oncol. 2012 Jun;48(6):560-6. 